\section{Osteology: a cornerstone of orthopaedic education}

Dear Sir,

The excellent article by F. F. A. IJpma, H. J. ten Duis, and T. M. van Gulik' was an enjoyable article to read and not only provides a historical overview of the works, study and examination of osteology by the masters of 16 th, 17th and 18th century, but also looks at the importance of these teachings to the evolution of orthopaedic surgery itself. After all, to quote, as the authors do, L. Heister and H. Ulhoorn: "To understand fracture healing, it is necessary to know the anatomy".

Over the past decade the teaching, and as a consequence the knowledge, of anatomy seems to have undergone a decline. This trend, even though it is hard to quantify and document, seems to be global. I often hear complaints by surgeons worldwide that today's medical graduates, entering a postgraduate training programme, lack the basic anatomical knowledge necessary to practice effectively and safely. This is even more important in surgical specialties. This trend seems to coincide, as the authors so rightly point out, with the introduction of new teaching technologies in anatomy which rely mostly on computer-generated models and medical imaging. Dissection, though, not only teaches anatomy but also how to respect human tissues. After all, anatomy and surgery should involve all senses, not only vision. This article reminds us that we should not re-invent the wheel, and that the tried and tested approach of our surgical forefathers should be respected.

Another important issue discussed is that of medical qualification. Today's open borders have allowed numerous medical doctors to migrate to different countries despite the heterogeneity of anatomy and medical teaching at different institutions and in different countries. In an era of discussion of pan-European validation and re-validation this will soon prove to be a major issue. Studying our medical history can help us solve this problem.

A. Papavasiliou BSC, MD, PhD, Consultant Orthopaedic Surgeon, Lecturer in Sport Injuries, Aristotle University of Thessaloniki, St Luke's Hospital,

Greece

REFERENCES

1. IJpma FFA, ten Duis HJ, van Gulik TM. Osteology: a cornerstone of orthopaedic education. Bone Joint 360 2012;1(6):2-7. 